\title{
DEVELOPMENT OF DIFFERENTIAL EQUATIONS AND THEIR SOLUTION USING THE SIMULINK MATLAB PROGRAM, WHICH CALCULATE THE SELF-SWINGING OF SYNCHRONOUS MACHINES WITH TRADITIONAL AND LONGITUDINAL- TRANSVERSE EXCITATION
}

\author{
Olimjon Toirov ${ }^{*}$, Allabergan Bekishev ${ }^{2}$, Sardor Urakov ${ }^{3}$, Utkir Mirkhonov $^{4}$ \\ ${ }^{1}$ Tashkent State Technical University, DSc, Prof. Head of the Department of Electrical machine, Tashkent, 100095, Uzbekistan \\ ${ }^{2}$ Tashkent State Technical University, Department of Electrical machine, Tashkent, 100095, Uzbekistan \\ ${ }^{3}$ Tashkent State Technical University, Department of Electrical machine, Tashkent, 100095, Uzbekistan \\ ${ }^{4}$ Bukhara Engineering Technological Institute, Department of Energy audit, Bukhara, 200117, Uzbekistan
}

\begin{abstract}
The article presents the differential equations of a synchronous generator in phase coordinates and in the coordinate system $(\mathrm{d}, \mathrm{q})$. In addition, differential equations of synchronous machines with longitudinal-transverse excitation and a block diagram based on these equations are given. The system of differential equations is solved by the operator method. On the basis of a system of differential equations using the Simulink Matlab program, a structural diagram was created and a graph of the self-swinging processes taking place in synchronous machines with conventional and longitudinal-transverse excitation was obtained. On the basis of the obtained graph, the processes of self-swinging of synchronous generators with traditional and longitudinal and transverse excitation are compared.
\end{abstract}

\section{Introduction}

The relevance of the topic lies in the fact that the determination of the static and dynamic stability of synchronous generators of medium and high power in laboratory conditions using these generators requires a lot of money and time. To save the necessary funds and time, it is advisable to conduct research on mathematical models of synchronous generators of medium and high power. In addition, the development of biaxial synchronous generators with magnetic axis control and their use in power plants is currently underway $[8,9]$.

The static and dynamic stability of synchronous generators with longitudinal-transverse excitation is a poorly studied area, and the study of this area is useful for studying the stability of generators by developing differential equations for synchronous generators with biaxial excitation.

To improve the performance and energy efficiency of synchronous machines, two coils are placed on the rotor at a distance of 90 degrees from each other. When we use a synchronous machine with biaxial excitation, it will give us the opportunity to control the direction of the magnetic flux vector, increase the static and dynamic stability of the machine and more effectively dampen the vibration of the rotor under alternating and shock loads [7].

The aim of the work is to study the effect of an additional excitation coil on the transverse axis on the self-swinging processes of synchronous machines with longitudinaltransverse excitation using a mathematical model and comparison with the self-swinging processes of a traditional synchronous generator.

\subsection{Construction of mathematical equations for traditional synchronous machines:}

When constructing differential equations for synchronous machines, we take into account the following assumptions:

1) the magnetic permeability of the steel core of the synchronous machine is infinite;

2) The stator and rotor magnetic fields are sinusoidally distributed along the air gap;

3 ) the phase windings are symmetrical;

4) Damping rods are symmetrical about the rotor axis. a)

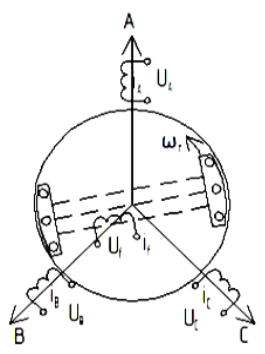

b)

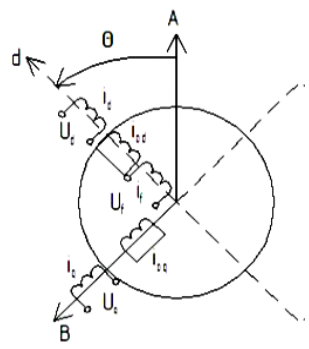

Fig 1.1. View of a salient-pole synchronous machine in a) phase coordinate system $(\mathrm{A}, \mathrm{B}, \mathrm{C})$ and b) rotor coordinate system (d, 
According to the stator equation, machine voltages (Fig. 1.1, a) are as follows [1]:

$$
\left.\begin{array}{l}
U_{A}=i_{A} r_{S}+\frac{d \Psi_{A}}{d t} ; \\
U_{B}=i_{B} r_{S}+\frac{d \Psi_{B}}{d t} ; \\
U_{C}=i_{C} r_{S}+\frac{d \Psi_{C}}{d t} ;
\end{array}\right\}
$$

where $i_{A}, i_{B}, i_{C}$ - instantaneous values of phase currents; $r_{S}$ is the active resistance of the stator phase winding; $\Psi_{\mathrm{A}}$, $\Psi_{\mathrm{B}}, \Psi_{\mathrm{C}}$ - flux linkage of phase stator windings.

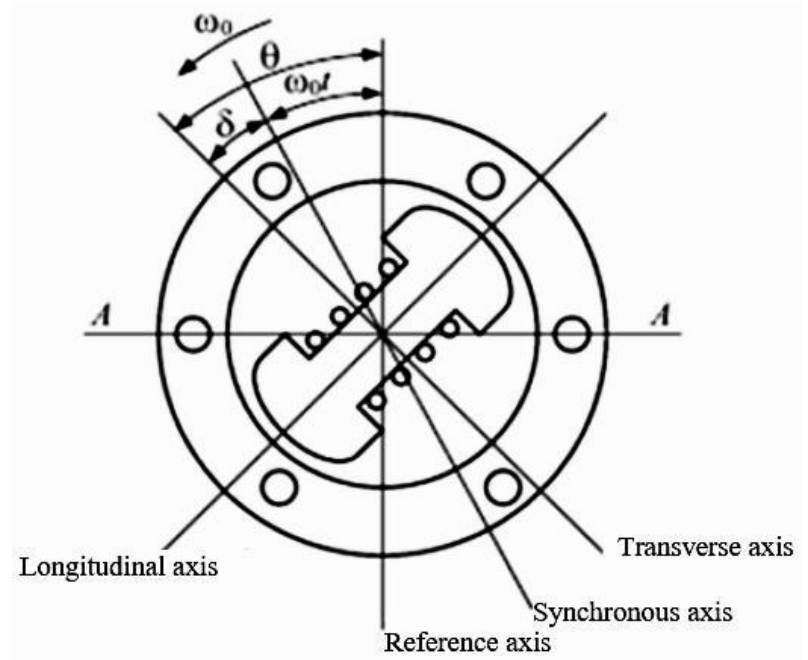

Fig 1.2. Synchronous generator motion parameters.

The stress equations along the axes $(d, q)$ for the damper winding and the excitation winding shown in (Fig1.1, b) are as follows [2]:

$$
\left.\begin{array}{c}
U_{f}=\frac{d \psi_{f}}{d t}+r_{f} i_{f} \\
0=\frac{d \psi_{D d}}{d t}+r_{D d} i_{D d} \\
0=\frac{d \psi_{D q}}{d t}+r_{D q} i_{D q}
\end{array}\right\}
$$

where $U_{f}$ is the voltage across the excitation winding; $i_{f}$, $i_{D d}, i_{D q}$ are the currents of the excitation and damper windings along the $\mathrm{d}$ and $\mathrm{q}$ axes; $r_{f}, r_{D d}, r_{D q}$ are the active resistances of the excitation and damper coils along the $\mathrm{d}$ and q axes; $\Psi_{f}, \Psi_{D d}, \Psi_{D q}$ - flux linkages of the excitation and damper coils. along the $\mathrm{d}$ and $\mathrm{q}$ axes.

Flux linkage system of equations of a synchronous machine [3]:

$$
\left.\begin{array}{l}
\Psi_{A}=L_{A} i_{A}+M_{A B} i_{B}+M_{A C} i_{C}+M_{A f} i_{f}+M_{A D d} i_{D d}+M_{A D q} i_{D q} ; \\
\Psi_{B}=M_{A B} i_{A}+L_{B} i_{B}+M_{B C} i_{C}+M_{B f} i_{f}+M_{B D d} i_{D d}+M_{B D q} i_{D q} ; \\
\Psi_{C}=M_{C A} i_{A}+M_{C B} i_{B}+L_{C} i_{C}+M_{C f} i_{f}+M_{C D d} i_{D d}+M_{C D q} i_{D q} ; \\
\Psi_{f}=M_{f A} i_{A}+M_{f B} i_{B}+M_{f C} i_{C}+L_{f} i_{f}+M_{f D d} i_{D d} \\
\Psi_{D d}=M_{D d A} i_{A}+M_{D d B} i_{B}+M_{D d C} i_{C}+M_{D d f} i_{f}+L_{D d} i_{D d} ; \\
\Psi_{D q}=M_{D q A} i_{A}+M_{D q B} i_{B}+M_{D q C} i_{C}+M_{D q f} i_{f}+L_{D q} i_{D q} ;
\end{array}\right\}
$$

where $L$ and $M$ - inductance and mutual inductance of different coils and $M_{A B}=M_{B A} ; M_{f A}=M_{A f} ; M_{D d A}=M_{A D d}$ and so on. $L_{f}$ is the inductance of the field coil; $L_{D d}, L_{D q}$ longitudinal and transverse inductance of the damper coil.
The inductance and mutual inductance of the phase windings is a periodic function of the angle between the longitudinal axis of the machine and the axes of phase A:

$$
\left.\begin{array}{l}
L_{A}=L_{0}+L_{2} \cos 2 \theta \\
L_{B}=L_{0}+L_{2} \cos \left(2 \theta+\frac{2 \pi}{3}\right) ; \\
L_{C}=L_{0}+L_{2} \cos \left(2 \theta+\frac{4 \pi}{3}\right) ; \\
M_{A B}=M_{0}+M_{2} \cos \left(2 \theta-\frac{2 \pi}{3}\right) ; \\
M_{A C}=M_{0}+M_{2} \cos \left(2 \theta+\frac{2 \pi}{3}\right) ; \\
M_{B C}=M_{0}+M_{2} \cos 2 \theta
\end{array}\right\}
$$

where $L_{0}=2 M_{0}$ and $L_{2}=M_{2}$, the coils are arranged symmetrically.

The mutual inductance between the phase windings and the excitation winding and the damper windings is as follows:

$$
\begin{aligned}
& M_{A f}=M_{A f d} \cos \theta ; \\
& M_{B f}=M_{A f d} \cos \left(\theta-\frac{2 \pi}{3}\right) ; \\
& M_{C f}=M_{A f d} \cos \left(\theta+\frac{2 \pi}{3}\right) ; \\
& M_{A D d}=M_{A D d} \cos \theta ; \\
& M_{B D d}=M_{A D d} \cos \left(\theta-\frac{2 \pi}{3}\right) ; \\
& M_{C D d}=M_{A D d} \cos \left(\theta+\frac{2 \pi}{3}\right) ; \\
& M_{A D q}=M_{A D q} \sin \theta ; \\
& M_{B D q}=M_{A D q} \sin \left(\theta-\frac{2 \pi}{3}\right) ; \\
& M_{B D q}=M_{A D q} \sin \left(\theta-\frac{2 \pi}{3}\right) ;
\end{aligned}
$$

where $M_{A f d}, M_{A D d}, M_{A D q}$ are the mutual inductances of the phase A coil and the excitation coil when the d, q axes and the damper contours coincide.

The reason for the difficulty in solving the system of equations 1.4 and 1.5 is that inductance and mutual inductance are a periodic function of the rotor state.

To exclude periodic coefficients, we write the system of equation in fixed coordinates $(\mathrm{d}, \mathrm{q})$ relative to the rotor:

$$
\left.\begin{array}{r}
U_{s d}=i_{s d} r_{s}+\frac{d \psi_{d}}{d t}-\omega_{s}\left(1-s_{M}\right) \psi_{q} ; \\
U_{s q}=i_{s q} r_{s}+\frac{d \psi_{q}}{d t}-\omega_{s}\left(1-s_{M}\right) \psi_{d} ; \\
u_{f}=\frac{d \psi_{f}}{d t}+r_{f} i_{f} \\
0=\frac{d \psi_{D d}}{d t}+r_{D d} i_{D d} \\
0=\frac{d \psi_{D q}}{d t}+r_{D q} i_{D q} \\
M_{\ni}=\frac{3}{2}\left(\Psi_{d} i_{s d}-\Psi_{q} i_{s q}\right) \\
J \omega_{s} \frac{d s_{M}}{d t}=M_{\mathrm{MECH}}-M_{E}
\end{array}\right\}
$$

where $s_{M}=s_{M}=\left(\omega_{r}-\omega_{s}\right) / \omega_{\mathrm{s}}$ - slip; $J$ - moment of inertia; $M_{M E C H}$ - mechanical torque on the shaft; $\mathrm{M}_{\mathrm{E}}$ - is the electromagnetic moment of the machine. 
The system of equation for the stator and rotor circuits is as follows:

$$
\left.\begin{array}{c}
\psi_{d}=L_{s d} i_{s d}+M_{A d}\left(i_{f}+i_{D d}\right) \\
\psi_{q}=L_{s q} i s_{q}+M_{A q} i_{D d} \\
\psi_{f}=L_{f} i_{f}+M_{A d} i_{d}+\frac{3}{2} M_{A d} i_{s d} \\
\psi_{D d}=L_{D d} i_{D d}+\frac{3}{2} M_{A d} i_{s d}+M_{A d} i_{f} \\
\psi_{D q}=L_{D q} i_{D q}+\frac{3}{2} M_{A q} i_{s q}
\end{array}\right\}
$$

where $L_{s d}, L_{s q}$ is the stator winding inductance along the longitudinal and transverse axes; $M_{A d}, M_{A q}$ - mutual inductance of any double circuit along the longitudinal and transverse axes of the machine.

\section{Mathematical equations of a synchronous generator with longitudinal-transverse excitation are constructed as follows:}

The differential equations of a synchronous machine with double axis excitation are constructed as follows:

$$
\left.\begin{array}{c}
U_{d}=r_{s} i_{d}+p \Psi_{d}-\Psi_{q} \omega \\
U_{q}=r_{s} i_{q}+p \Psi_{q}+\Psi_{d} \omega \\
U_{f d}=r_{f d} i_{f d}+p \Psi_{f d} \\
U_{f q}=r_{f q} i_{f q}+p \Psi_{f q} \\
0=r_{D d} i_{D d}+p \Psi_{D d} \\
0=r_{D q} i_{D q}+p \Psi_{D q} \\
M_{\ni}=\frac{3}{2}\left(\Psi_{d} i_{s d}-\Psi_{q} i_{s q}\right) \\
J p \omega=\Psi_{d} i_{q}-\Psi_{q} i_{d}-M_{\mathrm{MECH}}
\end{array}\right\}
$$

The flux linkage of the circuits of a synchronous machine with double excitation is calculated as follows:

$$
\begin{aligned}
& \Psi_{d}=x_{\sigma} i_{d}+x_{a d}\left(i_{d}+i_{f}+i_{D d}\right)=x_{\sigma} i_{d}+\Psi_{\delta d} \\
& \Psi_{q}=x_{\sigma} i_{q}+x_{a q}\left(i_{d}+i_{D q}\right)=x_{\sigma} i_{q}+\Psi_{\delta q} \\
& \Psi_{f d}=x_{\sigma f d} i_{f d}+x_{a d}\left(i_{d}+i_{f d}+i_{D d}\right)=x_{\sigma f d} i_{f d}+\Psi_{\delta d} \\
& \Psi_{f q}=x_{\sigma f q} i_{f q}+x_{a q}\left(i_{q}+i_{f q}+i_{D q}\right)=x_{\sigma f q} i_{f q}+\Psi_{\delta q} \\
& \Psi_{D d}=x_{\sigma D d} i_{D d}+x_{a d}\left(i_{d}+i_{f d}+i_{D d}\right)=x_{\sigma D d} i_{D d}+\Psi_{\delta d} \\
& \Psi_{D q}=x_{\sigma D q} i_{D q}+x_{a d}\left(i_{q}+i_{D d}\right)=x_{\sigma D q} i_{D q}+\Psi_{\delta q}
\end{aligned}
$$

From the system of equations 2.1 and 2.2 we find the flux linkage of the contours [4]:

$$
\left.\begin{array}{c}
\Psi_{d}=\left(x_{\sigma} i_{d}+\Psi_{\delta d}\right)=\frac{1}{p}\left(U_{d}+\Psi_{q} \omega-r_{s} i_{d}\right) \\
\Psi_{q}=\left(x_{\sigma} i_{q}+\Psi_{\delta q}\right)=\frac{1}{p}\left(U_{q}-\Psi_{d} \omega-r_{s} i_{q}\right) \\
\Psi_{f d}=\left(x_{\sigma f d} i_{f d}+\Psi_{\delta d}\right)=\frac{1}{p}\left(U_{f d}+r_{f d} i_{f d}\right) \\
\Psi_{f q}=\left(x_{\sigma f q} i_{f q}+\Psi_{\delta q}\right)=\frac{1}{p}\left(U_{f q}+r_{f q} i_{f q}\right) \\
\Psi_{D d}=\left(x_{\sigma D d} i_{D d}+\Psi_{\delta d}\right)=-\frac{1}{p} r_{D d} i_{D d} \\
\Psi_{D q}=\left(x_{\sigma D q} i_{D q}+\Psi_{\delta q}\right)=-\frac{1}{p} r_{D q} i_{D q}
\end{array}\right\}
$$

The difference between the systems of equations 1.6 and 2.1 is that in the system of equations 2.1 there is an equation for the excitation winding along the $\mathrm{d}$ and $\mathrm{q}$ axes, in the system of equations 1.6 there is no flux linkage - $\Psi_{f q}$. In the system of equations 2.1 , the flux linkage - $\Psi_{f q}$ for the second axis q is also specified.

There are two methods for solving differential equations of electrical machines: analytical and operator. The analytical method is mainly used when solving linear equations, while the operator method is widely used when solving nonlinear equations. The above differential equations of our synchronous generators are non-linear because of the saturation of the magnetic circuit.

Self-swinging is an electromechanical instability of a synchronous generator that goes out of synchronism due to a change in the rotation speed and deflection angle of the rotor during operation of the synchronous generator. Self-swinging of a synchronous generator can occur for 3 reasons [5]:

a) the presence of a large active resistance in the stator circuit;

b) the presence of an insensitive zone of the device for automatic regulation of excitation and its delayed response;

c) automatic regulation of excitation due to incorrect device settings.

Below is a block diagram for the system of equations 2.1 and 2.3 in the Simulink Matlab program to study the process of self-swinging of a synchronous generator with longitudinal-transverse excitation during incorrect adjustment of excitation control [6]:

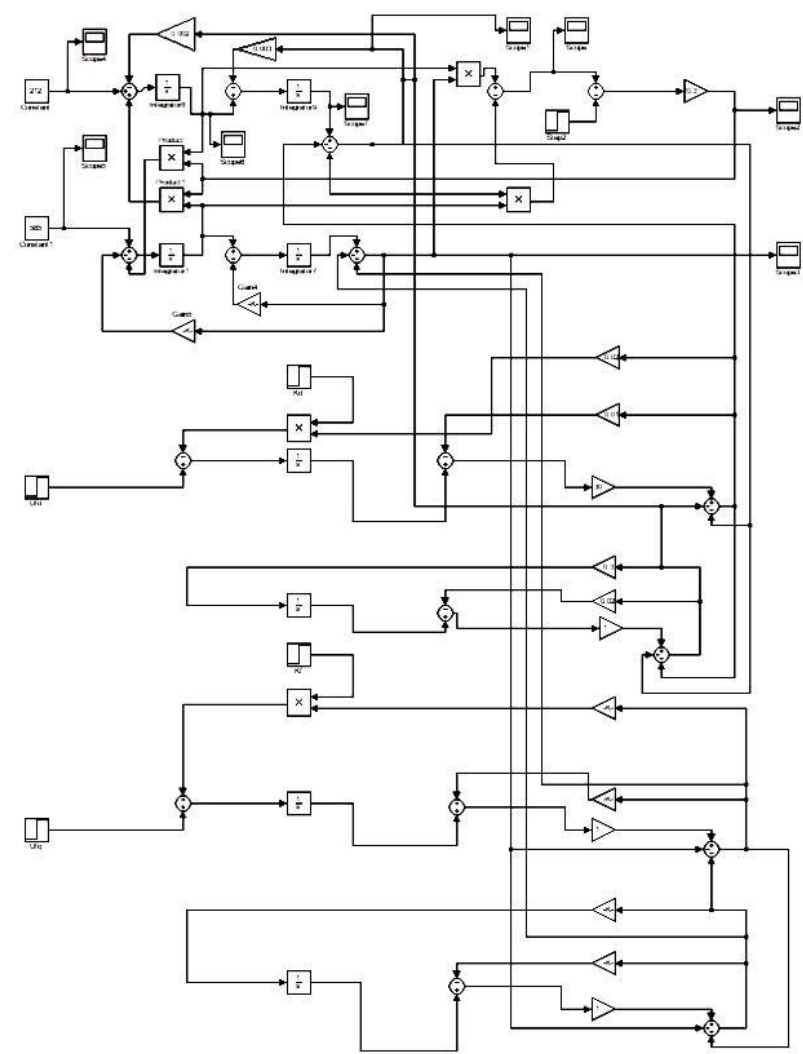

Fig 2.1. Block diagram in the Simulink Matlab program based on the system of equations 2.1 and 2.3 of a synchronous machine with longitudinal-transverse excitation. 

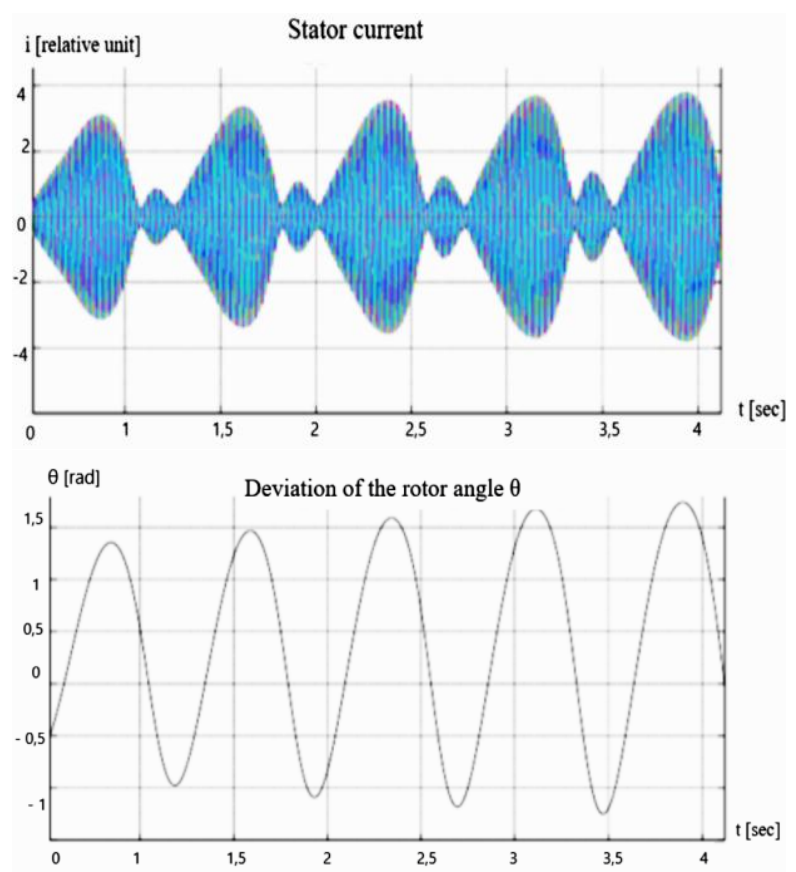

Fig 2.2. The graph of the self-swinging process with an incorrectly tuned device for automatic regulation of the excitation of synchronous generators with traditional excitation.
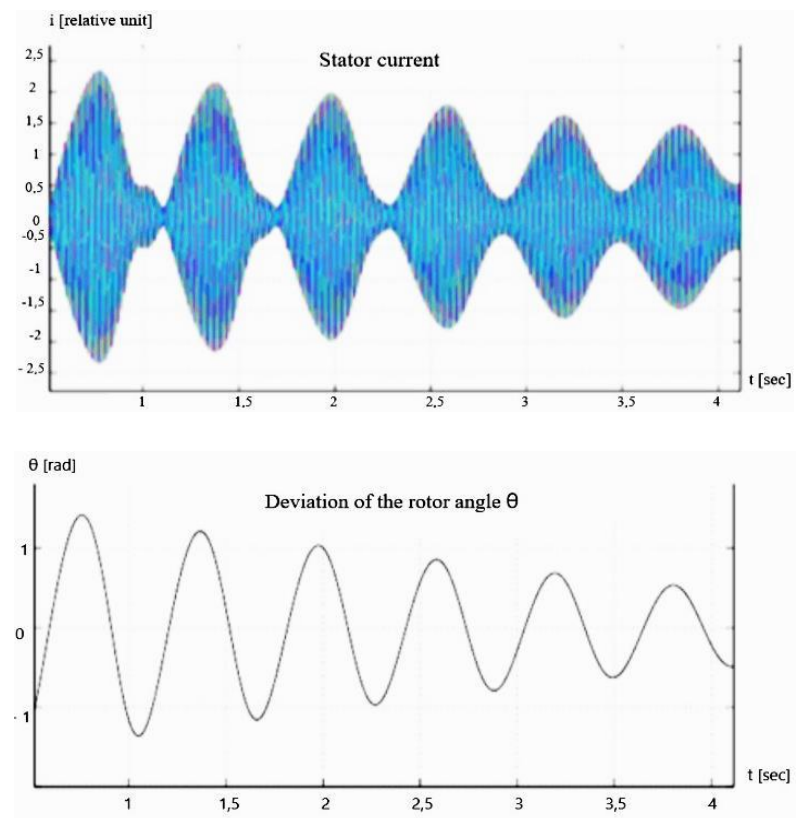

Fig 2.3. The graph of the self-swinging process with incorrect adjustment of the automatic excitation regulator of synchronous generators with longitudinal-transverse excitation

\section{Conclusion}

1. According to the results obtained (Fig.2.2), in a traditional synchronous generator, during incorrect tuning, the automatic regulator device excitation at the beginning of the self-swinging process, the stator current exceeds 4 times the nominal current and from the graph of the rotor deflection angle it can be seen that the traditional synchronous generator comes out out of synchronicity due to an increase in the amplitude of the rotor oscillation.
2. During the self-swinging of synchronous generators with longitudinal-transverse excitation with incorrect adjustment of the automatic excitation control device (Fig-2.3), the stator current increases 1.5 times and it was noticed that in a synchronous generator with longitudinaltransverse excitation due to a decrease in amplitude fluctuations in the rotor deflection angle, the selfswinging process decreases and turns into synchronism. 3. In synchronous generators with longitudinal-transverse excitation, static and dynamic stability is much higher than that of traditional synchronous generators due to the presence of additional excitation coils and good electromagnetic coupling of the stator and rotor and the possibility of changing the magnetization angle in them.

\section{References}

1. Kopilov, I.P. Matematicheskoe modelirovanie elektricheskix mashin : ucheb. dlya vuzov / I.P. Kopilov. - M. : Vissh. shk., 2001. - 327 s.

2. V.A. Denisov, M.N. Tret'yakova. Teoriya i perexodnix prosessi elektromagnitnix ustroystv i elektromexanicheskiy preobrazovateley energii. Elektronnoe uchebnoe posobie. FGBOU VPO "Tol'yattinskiy gosudarstvenniy universitet" 2014. - 108 $\mathrm{S}$

3. German-Galkin, S.G. Matlab\&Simulink. Proektirovanie mexatronnix sistem na PK / S.G. GermanGalkin. - SPb. : KORONA-Vek, 2008. - 368 s.

4. M.M. Fayziev, N.A. Kurbanov, A.B. Imomnazarov, B.S. Bobonazarov, A.E. Bekishev, Modelirovanie neyavnopolyusnnogo sinxronnogo generatora $\mathrm{v}$ Matlab. Moskva. Vestnik nauki i obrazovaniya 2017 №5(29) Tom1, str10-14.

5. Pirmatov N.B, Bekishev A.E, Kurbanov N.A. Modelirovaniya samoraskachivaniya sinxronnogo generatora $v$ srede Simulink Matlab. Tendensii i Perspektivi Razvitiya nauki i obrazovaniya $\mathrm{v}$ usloviyax globalizasii. -Pereyaslav-Xmel'niskiy. 2018 №42, str 585-589.

6. Toirov O.Z., Bekishev A.E., Taniev M.X. Issledovanie kolebatel'noy ustoychivosti sinxronnix generatorov $\mathrm{V}$ srede MATLAB // Problemi informatiki i energetiki. Tashkent, 2019. №5. - S. 70-77.

7. Pirmatov N.B., Axmatov M.G., Kamalov I.K. Issledovanie raboti sinxronnogo dvigatelya $\mathrm{S}$ vozbujdeniem po prodol'noy i poperechnoy osyam pri udarnoy nagruzke. Elektrichestvo. - Moskva, 2003. №2. S.64-65.

8. Xrushev Yu.V., Zapodovnikov K.I., Yushkov A.Yu.. Elektromexanicheskie perexodnie prosessi v elektroenergeticheskix sistemax. - Tomsk: Tomskogo politexnicheskogo universiteta. 2012. - S.160.

9. Dmitriy Bystrov, Toirov Olimjon, Mustafakulova Gulzoda, Yakubova Dilfuza, Fuzzy Systems for Computational Linguistics and Natural Language // NISS2020: Proceedings of the 3rd International Conference on Networking, Information Systems \& Security, March 2020, Morocco, Article No.: 54, P.1-3. https://doi.org/10.1145/3386723.3387873 